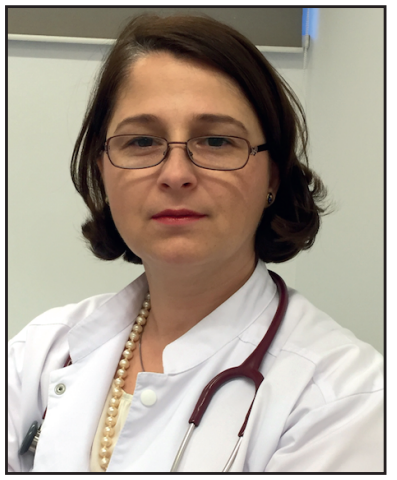

Camelia C. DIACONU ${ }^{\bowtie}$

International Secretary General of the Balkan Medical Union

President of the Romanian National Section

Secrétaire Général International de l'Union Médicale Balkanique

Présidente de la Section Nationale Roumaine

\section{COVID-19 AND HYPONATREMIA}

COVID-19 is an ongoing pandemic infectious disease determined by a new coronavirus, severe acute respiratory syndrome coronavirus (SARS-CoV-2). The severity spectrum of the clinical manifestations of the disease is variable, from almost asymptomatic cases to critical, fatal cases. The clinical manifestations usually include fever, dry cough, dyspnea, muscular symptoms, headache, sore throat, diarrhea, nausea/ vomiting, abdominal pain, fatigue, loss of smell or taste. The laboratory tests in patients with COVID-19 may reveal lymphopenia, increased aminotransferases, high lactate dehydrogenase, inflammatory syndrome (high C-reactive protein, erythrocyte sedimentation rate, fibrinogen), coagulation tests abnormalities. There are recent reports and small studies that reported an association between COVID-19 and hyponatremia (serum natrium $<135 \mathrm{mEq} / \mathrm{L}$ ) ${ }^{1}$. A few case reports described patients with COVID-19 with only symptoms of hyponatremia ${ }^{2}$. The etiology of hyponatremia in patients with COVID-19 is likely to be multifactorial and its early etiologic diagnosis may be extremely important for the evolution of the disease. For a correct diagnosis and treatment, measurement of plasma and urine osmolality is always necessary. An appropriate treatment of hyponatremia may be even more important in hospitalized patients with COVID-19, in whom an inadequate fluid and electrolyte management may increase the risk for respiratory and/or cardiac complications. What could be the causes of hyponatremia in COVID-19 patients?

\section{COVID-19 ET L'HYPO- NATREMIE}

Le COVID-19 est une maladie infectieuse pandémique en cours, déterminée par un nouveau coronavirus, le coronavirus du syndrome respiratoire aigu sévère (SARS-CoV-2). Le spectre de gravité des manifestations cliniques de la maladie est variable, allant des cas presque asymptomatiques aux cas critiques et mortels. Les manifestations cliniques comprennent généralement de la fièvre, une toux sèche, une dyspnée, des symptômes musculaires, des maux de tête, des maux de gorge, de la diarrhée, des nausées/vomissements, des douleurs abdominales, de la fatigue, une perte d'odorat ou de goût. Les tests de laboratoire chez les patients atteints de COVID-19 peuvent révéler une lymphopénie, une augmentation des aminotransférases, une lactate déshydrogénase élevée, un syndrome inflammatoire (protéine C-réactive élevée, vitesse de sédimentation érythrocytaire et fibrinogène élevés), des anomalies des tests de coagulation. Il existe des rapports récents et de petites études faisant état d'une association entre le COVID-19 et l'hyponatrémie (sérum natrium $<135 \mathrm{mEq} / \mathrm{L}$ ) ${ }^{1}$. Quelques rapports de cas décrivaient des patients atteints de COVID-19 avec uniquement des symptômes d'hyponatrémie 2 . L'étiologie de l'hyponatrémie chez les patients atteints de COVID-19 est probablement multifactorielle et son diagnostic étiologique précoce peut être très important pour l'évolution de la maladie. Pour un diagnostic et traitement corrects, la mesure de l'osmolalité plasmatique et urinaire est toujours nécessaire. Un traitement approprié de l'hyponatrémie peut être encore 
Hyponatremia may be due to the increased release of antidiuretic hormone $(\mathrm{ADH})$ because of gastrointestinal fluid losses (diarrhea, vomiting) or low oral fluid intake, but also to the syndrome of inappropriate ADH secretion (SIADH) induced by pneumonia, respiratory insufficiency or other comorbidities. SIADH is characterized by euvolemic hyponatremia (natrium $<135 \mathrm{mEq} / \mathrm{L}$ ), low serum osmolality (<280 mosmol/ $\left.\mathrm{Kg} \mathrm{H}_{2} \mathrm{O}\right)$, high urine osmolality (>100 mosmol/ Kg $\mathrm{H}_{2} \mathrm{O}$ ), increased urine natrium (>40 mmol/L), hypouricemia, in the absence of other causes of hyponatremia. Apart from pneumonia, that is frequent in patients with COVID-19, other causes of SIADH may be psychological and physical distress induced by the disease (with activation of the cortical neurons and increased hypothalamic secretion of $\mathrm{ADH}$ ), and also the cytokine storm (with lesions of the pulmonary tissue that can induce SIADH and/or the non-osmotic release of $\mathrm{ADH}$ stimulated by interleukin-6) ${ }^{3}$. The measurement of serum and urine osmolality will help the differential etiological diagnosis and will ensure a proper fluid and electrolyte management, to prevent further deterioration of these patients ${ }^{4}$.

The association of COVID-19 with hyponatremia needs to be further identified and explored. Until then, a careful approach may be that febrile patients presenting with hyponatremia should be isolated and tested for SARS-CoV-2 infection.

$$
\text { "No conflict of interest" }
$$

plus important chez les patients hospitalisés atteints de COVID-19, chez lesquels une gestion inadéquate des liquides et des électrolytes peut augmenter le risque de complications respiratoires et/ou cardiaques. Quelles pourraient être les causes de l'hyponatrémie chez les patients COVID-19? L'hyponatrémie peut être due à la libération accrue d'hormone antidiurétique (ADH) en raison de pertes de liquide gastro-intestinales (diarrhée, vomissements) ou d'un faible apport hydrique oral, mais aussi au syndrome de sécrétion inappropriée d'ADH (SIADH) induit par une pneumonie, une insuffisance respiratoire ou autres comorbidités. Le SIADH est caractérisé par une hyponatrémie euvolémique (natrium $<135 \mathrm{mEq} / \mathrm{L}$ ), une faible osmolalité sérique ( $<280 \mathrm{mosmol} / \mathrm{Kg} \mathrm{H}_{2} \mathrm{O}$ ), une osmolalité urinaire élevée ( $>100 \mathrm{mosmol} / \mathrm{Kg} \mathrm{H}_{2} \mathrm{O}$ ), une augmentation du taux urinaire natrium $(>40 \mathrm{mmol} / \mathrm{L})$, une hypo-uricémie, en l'absence d'autres causes d'hyponatrémie. Outre la pneumonie, qui est fréquente chez les patients atteints de COVID-19, d'autres causes de SIADH peuvent être la détresse psychologique et physique induite par la maladie (avec activation des neurones corticaux et augmentation de la sécrétion hypothalamique d'ADH), ainsi que la tempête de cytokines (avec lésions du tissu pulmonaire pouvant induire la SIADH et / ou la libération non osmotique d'ADH stimulée par l'interleukine-6)3. La mesure de l'osmolalité sérique et urinaire aidera au diagnostic étiologique différentiel et assurera une gestion adéquate des fluides et des électrolytes, pour éviter une détérioration supplémentaire de ces patients ${ }^{4}$.

L'association du COVID-19 avec l'hyponatrémie doit être davantage identifiée et explorée. D'ici là, une approche prudente peut être que les patients fébriles présentant une hyponatrémie doivent être isolés et testés pour une infection par le SARS-CoV-2.

\section{References}

1. Khan AA, Ata F, Munir W, Yousaf Z. Fluid replacement versus fluid restriction in COVID-19 associated hyponatremia. Cureus 2020;12(7):e9059.

2. Habib MB, Sardar S, Sajid J. Acute symptomatic hyponatremia in setting of SIADH as an isolated presentation of COVID-19. IDCases 2020;21:e00859.

3. Berni A, Malandrino D, Parenti G, Maggi M, Poggesi L, Peri A. Hyponatremia, IL-6, and SARS-CoV-2 (COVID-19) infection: may all fit together? Journal of Endocrinological Investigation 2020;43:1137-1139.

4. Aggarwal S, Garcia-Telles N, Aggarwal G, Lavie C, Lippi G, Michael Henry B. Clinical features, laboratory characteristics, and outcomes of patients hospitalized with coronavirus disease 2019 (COVID-19): early report from the United States. Diagnosis (Berl). 2020, 2:91-96. 